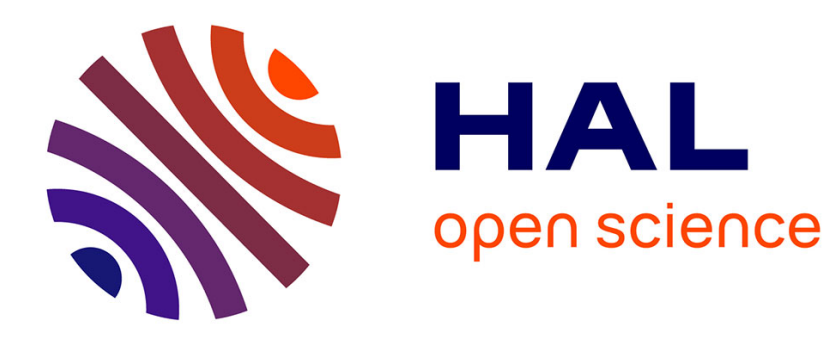

\title{
Recent Progress in the Theory of Bordoni-Type Relaxation Processes
}

\author{
W. Ulfert, A. Seeger
}

\section{To cite this version:}

W. Ulfert, A. Seeger. Recent Progress in the Theory of Bordoni-Type Relaxation Processes. Journal de Physique IV Proceedings, 1996, 06 (C8), pp.C8-207-C8-210. 10.1051/jp4:1996843 . jpa-00254651

\section{HAL Id: jpa-00254651 https://hal.science/jpa-00254651}

Submitted on 1 Jan 1996

HAL is a multi-disciplinary open access archive for the deposit and dissemination of scientific research documents, whether they are published or not. The documents may come from teaching and research institutions in France or abroad, or from public or private research centers.
L'archive ouverte pluridisciplinaire HAL, est destinée au dépôt et à la diffusion de documents scientifiques de niveau recherche, publiés ou non, émanant des établissements d'enseignement et de recherche français ou étrangers, des laboratoires publics ou privés. 


\title{
Recent Progress in the Theory of Bordoni-Type Relaxation Processes
}

\author{
W. Ulfert and A. Seeger \\ Max-Planck-Institut für Metallforschung, Institut für Physik, Heisenbergstr. 1, 70569 Stuttgart, Germany
}

\begin{abstract}
While it is well established by experiment that the relaxation processes in face-centred cubic metals discovered by P. G. Bordoni as well as related phenomena are due to the thermally activated formation of kink pairs on dislocation lines, the quantitative aspects of the theory as developed since Bordoni's discovery still reveal gaps or even inconsistencies. The paper reports on recent progress in solving the open problems.

An improved formula has been derived for the mean number of (thermal as well as geometrical) kinks on dislocation segments of length $L$ under the action of a homogeneous applied shear stress. It is shown how the change-over in the temperature dependence of the number of kink pairs, $n_{\mathrm{kp}}$, from the low-temperature $\exp \left(-2 H_{\mathrm{k}} / k T\right)$ law to the high temperature $\exp \left(-H_{\mathrm{k}} / k T\right)$ law depends on $L$, on the number of geometrical kinks $n_{\mathrm{g}}$, and on the applied stress. $H_{\mathrm{k}}$ is the formation energy of an isolated kink. The mean dislocation velocity, which is the essential quantity determining the relaxation time, shows a similar temperature dependence. The dependence of the relaxation time and strength of the relaxation process by kink-pair generation on internal stresses is discussed in some detail.
\end{abstract}

\section{Introduction}

In 1949 P. G. Bordoni [1] reported the observation of rather broad low-temperature internal-friction maxima in various cold-worked polycrystalline as well as monocrystalline face-centred cubic metals. Niblett and Wilks [2] established on $\mathrm{Cu}$ the existence of a second maximum at slightly lower temperatures than that discovered by Bordoni with similar characteristics, indications of which could be seen already in Bordoni's data [1]. Analogues of the Niblett-Wilks peak were subsequently discovered in the other fcc metals, too.

The Bordoni and the Niblett-Wilks maximum commonly denoted as "Bordoni relaxation" refers to the following now generally accepted interpretation: Dislocations running approximately along lowindex crystallographic directions overcome their Peierls barriers by the thermally activated generation of pairs of kinks of opposite sign. To a good approximation such a process may be characterized by a single temperature-dependent relaxation time $\tau(T)$. The peak temperature $T_{\mathrm{p}}$ is determined by

$$
2 \pi f \tau\left(T_{\mathrm{p}}\right)=1 \text {. }
$$

Different maxima are attributed to different types of dislocations with different $\tau(T)$.

The present paper reviews recent theoretical developments such as the simultaneous treatment of "geometrical" and "thermal" kinks (Sect. 2) and improvements in the calculation of the dislocation velocity and the relaxation time and strength (Sect. 3) of the kink-pair generation mechanism.

\section{Kinks in Thermal Equilibrium}

We consider a dislocation that is firmly anchored at two pinning points that are $n_{\mathrm{g}}$ Peierls valleys apart, where $a$ is the distance between neighbouring Peierls valleys. As length $L$ of the dislocation segment we denote the projection of the pinning-point separation on the Peierls valley direction. In addition to $n_{\mathrm{g}}$ geometrical kinks the segment may contain $n_{\mathrm{p}}$ pairs of kinks of opposite sign due to quantum and/or thermal fluctuations and the action of an effective stress $\sigma$, which throughout this paper is taken as homogeneous. The numbers of positive or negative kinks in the segment, $n_{+}$and 
$n_{-}$, are then given by

$$
n_{+}=n_{\mathrm{p}}, \quad n_{-}=n_{\mathrm{g}}+n_{\mathrm{p}}
$$

(For definitness the sign of the geometrical kinks has been chosen as negativ.) From now on we assume that the total number of kinks in the segment, $n$, obeys $\left(w_{\mathrm{k}}=\right.$ kink width)

$$
n=\left(n_{\mathrm{g}}+2 n_{\mathrm{p}}\right) \ll L / w_{\mathbf{k}} .
$$

The (approximate) soliton properties of kinks [3] allow us to treat positive and negative kinks as one-dimensional gases of non-interacting indistinguishable particles that are characterized by spatial coordinates $q_{j}$, momenta $P_{j}$, kink formation energy $H_{\mathrm{k}}$, and kink mass $m_{\mathrm{k}}$, and that are confined to the (one-dimensional) "volume" $L$. Then we obtain following partition function

$$
Z_{n_{\mathrm{g}}, n_{\mathrm{p}}}(L)=Z_{0,0}(L) \frac{Z_{+}^{n_{+}} Z_{-}^{n_{-}}}{n_{+} ! n_{-} !}
$$

Here $Z_{0,0}(L)$ denotes the partition function of a kink-free dislocation segment of length $L$ and

$$
Z_{+,-}=\frac{L_{+,-}^{*}}{\lambda_{\mathrm{k}}} \exp \left(S_{\mathrm{k}} / k_{\mathrm{B}}\right) \exp \left(-H_{\mathrm{k}} / k_{\mathrm{B}} T\right)
$$

$k_{\mathrm{B}}$ is Boltzmann's constant, and (with $h=$ Planck's constant)

$$
\sqrt{2 \pi} \lambda_{\mathrm{k}}:=h\left(m_{\mathrm{k}} k_{\mathrm{B}} T\right)^{-1 / 2}
$$

is the de Broglie wavelength attributable to kinks of mass $m_{\mathrm{k}}$ and kinetic energy $k_{\mathrm{B}} T / 2$. The quantity

$$
L^{*}:=\sqrt{L_{+}^{*} L_{-}^{*}}:=\left[\int_{0}^{L} \int_{0}^{L} \exp \left\{-\frac{V_{+}\left(q_{+}\right)+V_{-}\left(q_{-}\right)}{k_{\mathrm{B}} T}\right\} \mathrm{d} q_{+} \mathrm{d} q_{-}\right]^{1 / 2},
$$

where $V_{+,-}\left(q_{+,-}\right)$is the potential energy of positive or negative kinks due to, say, the effective stress $\sigma$, may be called the effective segment length $\left(L^{*}=L\right.$ if $\left.V(q) \equiv 0\right)$. The kink entropy $S_{\mathrm{k}}=S_{\mathrm{k}}(T)$ arises from the change of the vibration spectrum of a dislocation line upon the introduction of a kink [3]. For the present purpose it suffices to record the "high-temperature approximation"

$$
S_{\mathrm{k}}^{\mathrm{ht}}=k_{\mathrm{B}} \ln \left[h \sqrt{H_{\mathrm{k}} / m_{\mathrm{k}}} / w_{\mathrm{k}} k_{\mathrm{B}} T\right],
$$

In thermal equilibrium the probability that a dislocation segment with $n_{\mathrm{g}}$ geometrical kinks contains $n_{\mathrm{p}}$ kink pairs is

$$
p\left(n_{\mathrm{p}} ; n_{\mathrm{g}}, T\right)=Z_{n_{\mathrm{g}}, n_{\mathrm{p}}}(L) / \sum_{r=0}^{r_{\max }} Z_{n_{\mathrm{g}}, r}(L) .
$$

Hence the average number of kink pairs is given by

$$
\bar{n}_{\mathrm{p}}=\bar{n}_{\mathrm{p}}\left(n_{\mathrm{g}}, T\right)=\sum_{r=1}^{r_{\max }} r p\left(r ; n_{\mathrm{g}}, T\right) .
$$

Under almost all conditions of practical importance we may extend the summations in $(9,10)$ to infinity and express the resulting infinite series in terms of the modified Bessel function $I_{n_{g}}(\xi)$ of the argument $\xi:=2 \sqrt{Z_{+} Z_{-}}$. The final result is

$$
\vec{n}_{\mathrm{p}}=\left(\frac{\xi}{2}\right) \frac{\mathrm{I}_{n_{\mathrm{g}}+1}(\xi)}{\mathrm{I}_{n_{\mathrm{g}}}(\xi)}=\left(\frac{\xi}{2}\right) \frac{\mathrm{d}}{\mathrm{d} \xi}\left[\ln \mathrm{I}_{n_{\mathrm{g}}}(\xi)\right]-\frac{n_{\mathrm{g}}}{2} \quad \text { with } \quad \xi=2 \frac{L^{*}}{\lambda_{\mathrm{k}}} \exp \left(\frac{S_{\mathrm{k}}}{k_{\mathrm{B}}}\right) \exp \left(-\frac{H_{\mathrm{k}}}{k_{\mathrm{B}} T}\right) .
$$

In (11) the influence of the geometrical kinks appears in the order of the Bessel functions, whereas the other parameters (temperature, segment length, stress) are contained in their argument.

Making use of well-known properties of modified Bessel functions, we obtain from (11) the hightemperature limit $\left[\xi \gg\left(n_{\mathrm{g}}+1\right)\right]$

$$
\bar{n}_{\mathrm{p}}=\left(\xi-n_{\mathrm{g}}\right) / 2=\left(L^{*} / \lambda_{\mathrm{k}}\right) \exp \left(S_{\mathrm{k}} / k_{\mathrm{B}}\right) \exp \left(-H_{\mathrm{k}} / k_{\mathrm{B}} T\right)-n_{\mathrm{g}} / 2,
$$

and in contrast to earlier work the intuitively correct low temperature approximation $\left(\xi<n_{\mathrm{g}}+1\right)$

$$
\bar{n}_{\mathrm{p}}=\frac{\xi^{2}}{4\left(n_{\mathrm{g}}+1\right)}=\frac{1}{\left(n_{\mathrm{g}}+1\right)}\left(L^{*} / \lambda_{\mathrm{k}}\right)^{2} \exp \left(2 S_{\mathrm{k}} / k_{\mathrm{B}}\right) \exp \left(-2 H_{\mathrm{k}} / k_{\mathrm{B}} T\right)
$$

In the remainder of this section we discuss, on the basis of $(7)$, the relationship of the effective segment length $L^{*}$ to the "true segment length" $L$ (for the precise meaning of $L$ see above). Taking 
into account the so-called Peierls potential of the second kind [3], i.e. the periodic variation of the kink energy along an otherwise straight dislocation line, gives always $L^{*} / L<1$. However, in metals (in contrast to valence-bond crystals) this effect is small enough to be neglected in the present context. More important are stresses $\sigma$ such as the applied stress or long-range internal stresses that may be treated as homogeneous.

The force exerted on a kink of height $a$ by the stress component $\sigma$ in the glide system of a dislocation of strength $b$ is $\pm a b \sigma$, depending on the sign of the kink. Inserting

into (7) gives us

$$
V_{+,-}(q)= \pm a b \sigma(q-L / 2)
$$

$$
L^{*} / L=\frac{2 k_{\mathrm{B}} T}{a b \sigma L} \sinh \left(\frac{a b \sigma L}{2 k_{\mathrm{B}} T}\right) \text {. }
$$

From (15) we see that homogeneous stresses always lead to $L^{*}>L$ and hence to an increase of the density of kink pairs, $\rho_{\mathrm{p}}=n_{\mathrm{p}} / L$. The stress causes positive and negative kinks to accumulate at opposite ends of the dislocation segment, as may be seen from the following expressions for the linear densities of positive and negative kinks, $\rho_{+}(q)$ and $\rho_{-}(q)$ :

$$
\rho_{+,-}(q)=\frac{\exp \left[-V_{+,-}(q)\right]}{L^{*}} \bar{n}_{+,-}
$$

\section{Dislocation Velocity and Relaxation Time}

For the probability $p_{r}$ that there are $r$ kink pairs per segment in an ensemble of dislocation segments of length $L$ lying approximately parallel to a Peierls valley direction, we may write down the rate equations

$$
\mathrm{d} p_{r} / \mathrm{d} t=\left(\Gamma_{\mathrm{k}} L p_{r-1}-p_{r} / \tau_{r}\right)-\left(\Gamma_{\mathrm{k}} L p_{r}-p_{r+1} / \tau_{r+1}\right) \quad(r=0,1,2,3 \ldots),
$$

where $\Gamma_{k}$ is the net rate of kink-pair formation per unit dislocation length [3] (Within the framework of the Klein-Kramers theory Mann [5] derived $\left.\Gamma_{\mathrm{k}}=2 v_{\mathrm{k}}\left(\xi / 2 L^{*}\right)^{2}\right)$. With $p_{-1}=0$ and $1 / \tau_{0}=0$ the stationary solution of (17) has to satisfy the difference equation

$$
\Gamma_{\mathbf{k}} L p_{r-1}-p_{r} / \tau_{r}=0 \quad(r=1,2,3 \ldots)
$$

Hence the stationary solution of (18) is given by

$$
p_{r}=p_{0}\left(\Gamma_{\mathrm{k}} L\right)^{r} \prod_{j=1}^{j=r} \tau_{j} \quad \text { with } \quad \sum_{r=0}^{r_{\max }} p_{r}=1 .
$$

The lifetime $\tau_{r}$ of a kink pair on a dislocation line containing $r$ kink pairs is limited either by the "absorption" (trapping) of the kinks at the end-points of the dislocation segment or by the annihilation of kinks of opposite sign. Either process involves the drift velocity $v_{\mathrm{k}}=a b \sigma D_{\mathrm{k}} / k_{\mathrm{B}} T$ that a kink requires under the influence of the effective shear stress. $D_{\mathrm{k}}$ is the diffusivity of kinks moving along the dislocation line. The kink-pair lifetime can be expressed by

$$
1 / \tau_{r}=2 r(r+1) v_{\mathrm{k}} / L \text {. }
$$

In (20) the $r^{2}$-term describes the kink annihilation and the $r$-term the kink absorption. According to the kink description of the dislocation motion a dislocation segment of length $L$ containing $r$ kink pairs moves with a mean velocity.

$$
v_{\mathrm{d}}=2 v_{\mathrm{k}} \mathrm{ra} / L
$$

In the same approximation as above by extending the summations to infinity the average steady-state velocity of an ensemble of dislocation segments of length $L$ is thus given by

$$
\bar{v}_{d}=\frac{2 a}{L} v_{\mathrm{k}} \sum_{r=1}^{\infty} r p_{r}=\frac{a}{L} v_{\mathrm{k}} \frac{x \mathrm{I}_{2}(x)}{\mathrm{I}_{1}(x)} \quad \text { with } \quad x:=2 L \sqrt{\frac{\Gamma}{2 v_{\mathrm{k}}}} .
$$

Eq. (22) replaces an intuitive interpolation formula [4] that was based on the limiting cases $v_{\mathrm{d}}=$ $a L \Gamma_{\mathrm{k}} / 2(x \ll 1$, low temperatures, kink-pair annihilation negligible $)$ and $v_{\mathrm{d}}=a\left(2 v_{\mathrm{k}} \Gamma_{\mathrm{k}}\right)^{1 / 2}(x \gg 1$, high temperatures, pair annihilation dominant).

The determination of the relaxation function of the kink-pair relaxation mechanism requires the time-dependent solution of the rate equation (17), a topic that will be treated elsewhere. Here we make the approximation that the mean value of the kink-pair life times defined in eq. (20) is always shorter than the relaxation time $\tau_{\mathrm{kp}}$ (which indeed turns out to be fulfilled). Then we are allowed to deal with the steady state approximation of the dislocation velocity $v_{\mathrm{d}}$ given in eq. (22). 


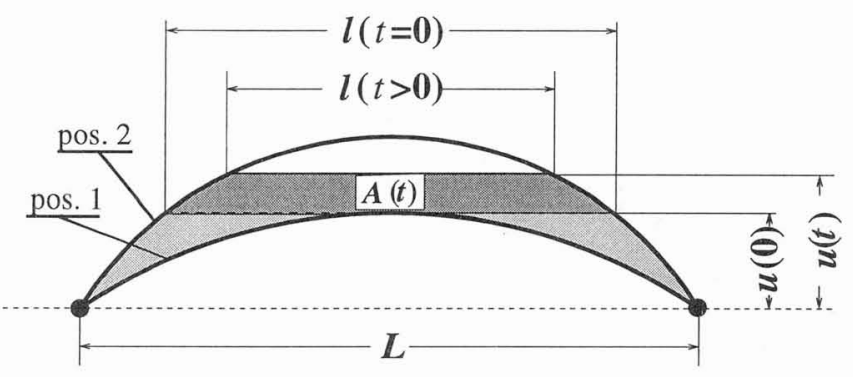

Figure 1: Dislocation line bowed out under the action of internal $\left(\sigma_{\mathrm{i}}\right.$, pos. 1$)$ and external $\left(\sigma_{\mathrm{e}}\right.$, pos. 2$)$ stresses.
The relaxation mechanism of a dislocation segment of length $L$ is explained in fig. 1: At time $t \leq 0$ the segment may be swept out to its equilibrium configuration according to the effective internal stress $\sigma_{\mathrm{i}}$ (pos. 1). This equilibrium configuration is determined by the balance between the repulsion between the kinks and the force $a b \sigma_{\mathrm{i}}$ exerted on the kinks. For its determination we use the so-called string approximation, characterized by a line tension $S_{\mathrm{d}}$. At $t=0$ a homogeneous external stress $\sigma_{\mathrm{e}}$ may be applied. The new equlibrium configuration according to the total stress $\sigma=\sigma_{\mathrm{i}}+\sigma_{\mathrm{e}}$ is denoted with pos.2. Before the displacement $u$ of the centre of the

dislocation segment increases by kink-pair formation the existing kinks will drift in direction of the dislocation line towards pos. 2. By this they left behind a straight part on the dislocation segment of length $l(0)$. The light grey area in fig. 1 which is swept out by this drift process do not contribute to the relaxation strength in low-frequency experiments which are considered in our case. In the course of time the slower process of kink-pair formation becomes active and the displacement $u$ increases, leaving behind the dark-grey area $A(t)$. Using a quadradic approximation of the dislocation line we get for the time evolution of $A$

$$
A(t)=\frac{1}{12}\left(\frac{b \sigma}{S_{\mathrm{d}}}\right)\left[\left(L \sqrt{\frac{\sigma_{\mathrm{e}}}{\sigma}}\right)^{3}-(l(t))^{3}\right] \quad \text { with } u(l)=\frac{b \sigma}{8 S_{\mathrm{d}}}\left(L^{2}-l^{2}\right) .
$$

Following Seeger [4] we get for time $t$ needed to increase the displacement $u$ from $u(0)$ to $u(t)$

$$
t=\int_{u(0)}^{u(t)} \frac{\mathrm{d} u}{v_{\mathrm{d}}}=\frac{b \sigma}{4 S_{\mathrm{d}}} \int_{l(t)}^{L \sqrt{\sigma_{\mathrm{e}} / \sigma}} \frac{l^{\prime} \mathrm{d} l^{\prime}}{v_{\mathrm{d}}\left(\sigma_{\mathrm{i}}, \sigma_{\mathrm{e}}, l^{\prime}\right)} .
$$

In eq. (24) we have to keep in mind that the effective force acting on kinks result on the internal and external stresses and the kink-kink repulsion. We can account for this by setting

$$
v_{\mathrm{k}}=a b \sigma \frac{D_{\mathrm{k}}}{k_{\mathrm{B}} T} \frac{l^{2}}{L^{2}}
$$

Then using the low-temperature approximation (LT) $v_{\mathrm{d}}=a l^{\prime} \Gamma_{\mathrm{k}} / 2$ and the high-temperature approximation (HT) $v_{\mathrm{d}}=a \sqrt{2 v_{\mathrm{k}} \Gamma_{\mathrm{k}}}$ we get following approximations for $A(t)$

$$
\begin{gathered}
A^{\mathrm{LT}}(t)=\frac{1}{12}\left(\frac{b \sigma_{\mathrm{e}}}{S_{\mathrm{d}}}\right) L^{3} \sqrt{\frac{\sigma_{\mathrm{e}}}{\sigma}}\left[1-\frac{1}{\left(t / 3 \tau^{\mathrm{LT}}+1\right)^{3}}\right] \quad \text { with } \quad \tau^{\mathrm{LT}}=\frac{L k_{\mathrm{B}} T}{12 a^{2} S_{\mathrm{d}} D_{\mathrm{k}}}\left(\frac{2 v_{\mathrm{k}}}{\Gamma_{\mathrm{k}}}\right) \sqrt{\frac{\sigma}{\sigma_{\mathrm{e}}}}, \\
A^{\mathrm{HT}}(t)=\frac{1}{12}\left(\frac{b \sigma_{\mathrm{e}}}{S_{\mathrm{d}}}\right) L^{3} \sqrt{\frac{\sigma_{\mathrm{e}}}{\sigma}}\left[1-\exp \left(-\frac{t}{\tau^{\mathrm{HT}}}\right)\right] \quad \text { with } \quad \tau^{\mathrm{HT}}=\frac{L^{2} k_{\mathrm{B}} T}{24 a^{2} S_{\mathrm{d}} D_{\mathrm{k}}} \sqrt{\frac{2 v_{\mathrm{k}}}{\Gamma_{\mathrm{k}}}}
\end{gathered}
$$

We see that only the high-temperature relaxation is of Debye type with a stress independent relaxation time $\tau^{\mathrm{HT}}$. The non-Debye relaxation at low temperatures is governed by a relaxation time $\tau^{\mathrm{LT}} \propto \sqrt{\left(\sigma_{\mathrm{i}}+\sigma_{\mathrm{e}}\right) / \sigma_{\mathrm{e}}}$. With $\sqrt{\Gamma / v_{\mathrm{k}}}=\xi / \sqrt{2} L^{*}[5]$ the temperature dependence of $\tau^{\mathrm{LT}}$ and $\tau^{\mathrm{HT}}$ are in essential the same as given in [4]. Since the relaxation strength $\Delta$ is proportional to $A_{\max } / L \sigma_{\mathrm{e}}$ in both cases $\Delta^{\mathrm{LT}, \mathrm{HT}} \propto L^{2} \sqrt{\sigma_{\mathrm{e}} /\left(\sigma_{\mathrm{i}}+\sigma_{\mathrm{e}}\right)}$ hold.

\section{References}

[1] P. G. Bordoni, Ric. Sci. 19, 851-862 (1949).

2] D. H. Niblett and J. Wilks, Phil. Mag. 1, 415-427 (1956).

3] A. Seeger and P. Schiller, Physical Acoustics, Vol. III A (ed. by W. P. Mason), 361-495, Academic Press, New York and London (1966).

[4] A. Seeger, J. Physique 42, C5-201-C5-226 (1981).

[5] E. Mann, phys. stat. sol. (b) 111, 541-553 (1982). 